\title{
Design in the Authentication and Billing System Based on Radius and 802.1x Protocol
}

\author{
Chaoyi Chen ${ }^{1, a}$, Jianyong Zhang ${ }^{2, b}$, Junli Liur, \\ ${ }^{1,2,3}$ Teaching and Training Department, Tianjin Institute of Software \\ Engineering, \\ Tianjin, China, 300387 \\ aemail:ccy_home@hotmail.com, ${ }^{b}$ email:1023930164@qq.com, ${ }^{c}$ email: \\ jlltj2010@163.com
}

\begin{abstract}
.
With the rapid development of internet, the efficiency and security of authentication and billing system is attracting more and more attention. This paper proposed an advanced authentication and billing solution by integrating the advantages of radius and $802.1 \mathrm{x}$ protocol for the consideration of internet performance and management request after comparing the feature of PPPoE, Radius and $802.1 x$ protocols. In the practical application, the solution this paper put forward reduces the network overhead and improves the network performance so that can prove it has an outstanding actual effect.

Key words: Billing system ; Authentication system; 802.1x protocol; Radius protocol

\section{Introduction}

With the fast development of internet, people can enjoy more and more pleasure and convenient from internet. Meanwhile, lots of problems were exposed such as the security, efficiency and management problem. It is necessary to have study with how to manage the internet and allocate resource using appropriate protocol.

Lots of domestic and international experts studied on this area. Ping Ding considered that the $802.1 \mathrm{x}$ misses its goals in access control denial of service (DoS) attacks and propose a Central Manager (CM) not only to take the responsibility of an authentication server, but also to add functionality to prevent denial of service attacks[1]; Pascal Urien and Guy Pujolle introduced smart cards ensuring strong authentication in IP networks, according to the extensible authentication protocol (EAP) [2]; Fanbao Liu and Yumeng Feng have a study on the security problem of PPPoE network[3]; Gaurav Sharma made use of currency like hash chain tokens in order to identify an efficient authentication and billing mechanism for faster handoffs in WLANs based on the IEEE 802.1x specification[4]. This paper not only compares the advantages and disadvantages between different internet protocols but also propose a solution integrate both of
\end{abstract}


the advantage of $802.1 \mathrm{x}$ and radius in order to the network protocol overhead and improve the network performance.

\section{Common authentication protocols}

RADIUS (Remote Authentication Dial In User Service) protocol is a sort of C/S structure protocol with advantages of simple, clear, stable, open and so on. It was put forward by Livingston Company with the purpose of authentication and billing for dial user and then developed to an general authentication protocol. Radius has widespread use so that can support the business of ADSL, IP telephone, VPDN and etc. Radius has stronger billing function using client and server model. The client-side is Network Access Server (NAS) and the serviceside is for receiving and saving the proceeding from NAS billing data. The communication protocol between them uses a simple account request packet to achieve communication between the client-side to server-side.

PPPoE protocol was developed by Redback Company and RouterWare Company after 1998. PPPoE protocol start PPP connect by establish a ethernet bridge. Using the PPPoE protocol, service providers can achieve user administration in internet. There are differences between PPP protocol and ethernet technology in essence, PPP protocol needs to be re packaged into an ethernet frame, so the encapsulation efficiency is very low and it have a great impact on network performance.

IEEE802.1x protocol is a protocol based on Client/Server visit controlling and authentication. Supplicants System, Authentication system and Authentication Server System is main parts of IEEE802.1x protocol. The advantages of $802.1 \mathrm{x}$ is that it can reduce the network building cost because of the lower request for equipment and has a good expansibility [5]. 802.1x can carry out billing based on the time but not easy to billing by users' network flow rate.

\section{System design}

\section{A. System analysis}

Authentication and billing system should have function of controlling users' access and make sure just the users with authentication can use the internet. When users use the internet have to bind the IP address, MAC address, switch port and VLAN ID with user account. The administrator not only can have the system function of billing management and user administration but also have the function of user self inquiry service, administrator accounting decentralized administration and telnet management. Meanwhile the system could solve the collision and stolen problem of IP address so that can improve the system security. 


\section{B. System policy}

Network billing policy using two modes of flow rate and time, users can choose it depend on themselves. The Authentication using 802.1x add radius solution that every subnet users information send to radius server from NAS (Network Access Server). When identity verification passed, DHCP server would distribute IP address to users and allow them using the network resource. In the user access side, solution uses the switch with $802.1 \mathrm{x}$ protocol technique to administrate the user visit port. User have to setup a client program to achieve the user internet authentication. Server side uses the AAA authentication server based on the radius protocol and setup HTTP service, data base system and DHCP server to avoid the IP address collision.

\section{Billing system software structure}

When the authentication completes, using access charge judgment module to determine whether the user has sufficient amount of access network ( account of the amount $>0$ ). The module will keep the user network connection or disconnection according to the actual situation ( by controlling the controlled port state ). When the server enforced disconnect user network connection, or user self disconnect network, the information of the flow and the time of the network will be written into the database automatically. Start billing strategy modular at regular time, to calculate the network communication cost of the user in the timing range, and to modify the account balance in database. Billing system software structure is shown in figure 1 . 


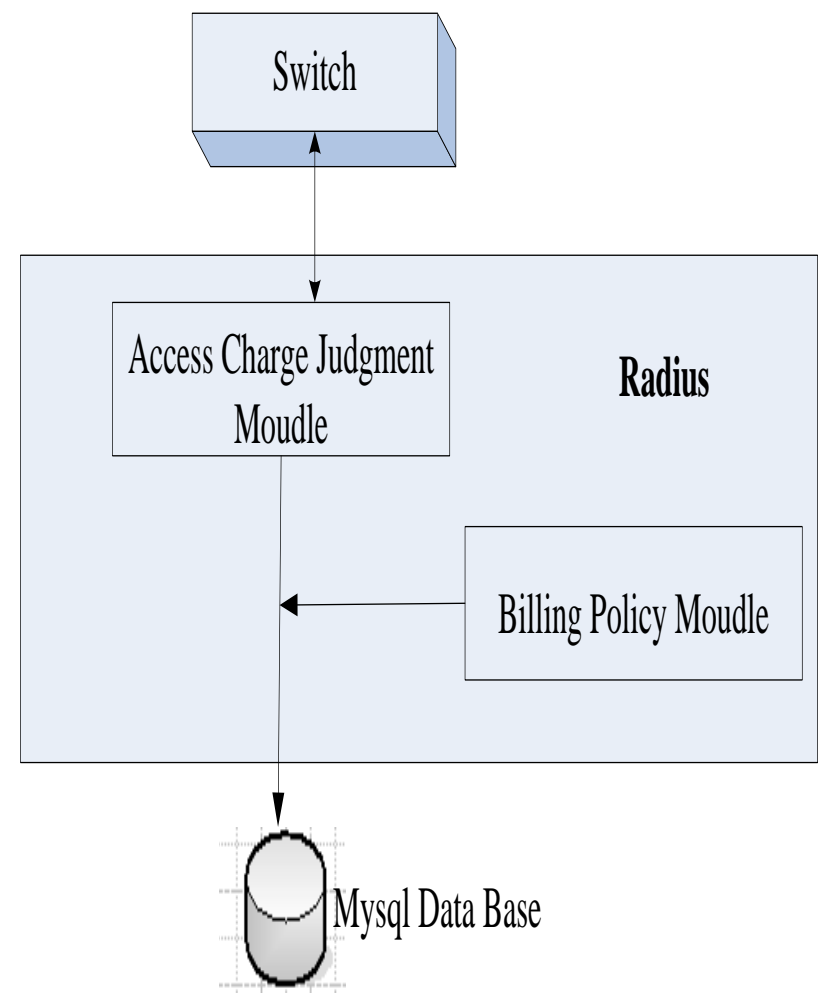

Figure 1: Billing system software structure

D. The network architecture of authentication and billing management system

The user connected switches through the client. The switch send the user request information to the Radius server through the uncontrolled port for authentication. If the authentication passed, it will open a controlled port of the logical port of the switch. Then the user could communicate through the controlled port. The switch will send the traffic and time information to Radius server at any time. This will be the raw data of billing. The network architecture of authentication and billing management system is shown in figure 2 . 


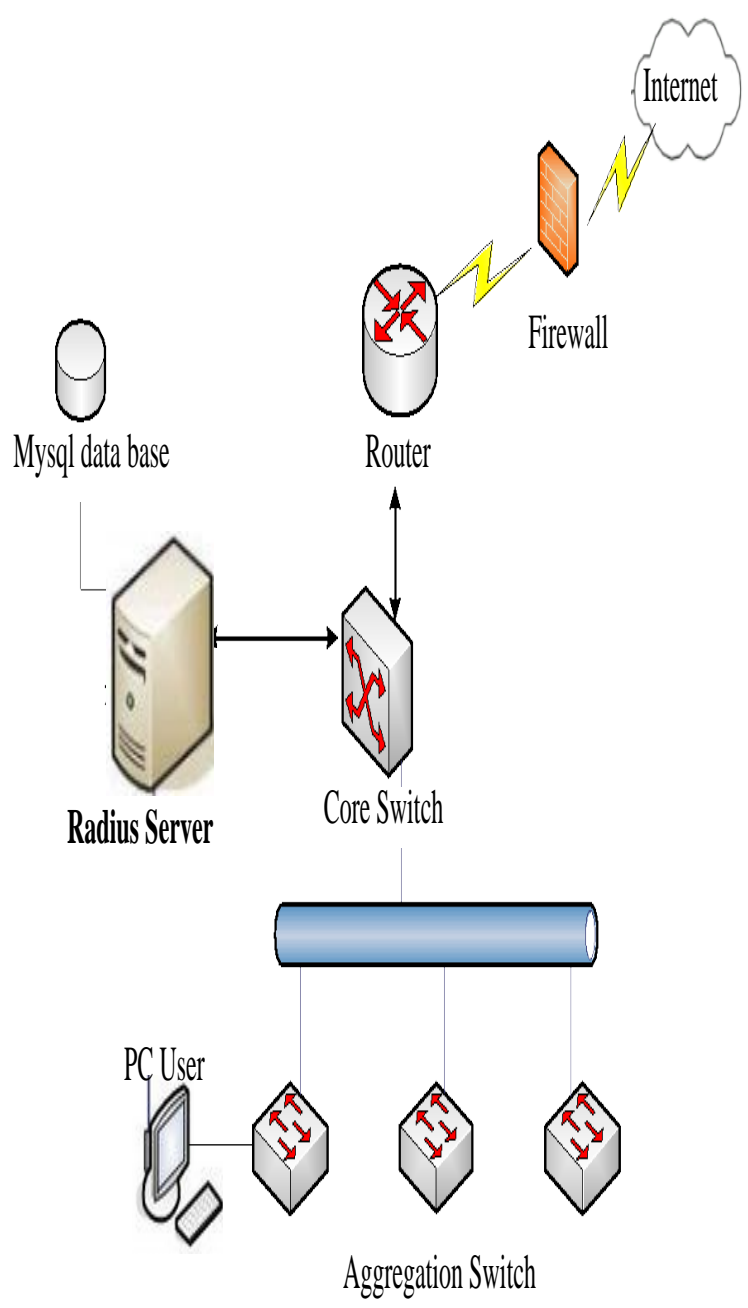

Figure 2: The network architecture of authentication and billing management system

\section{Practical application}

This paper uses Tianjin Institute of Software Engineering (TJISE) as a practical application case to indicate the performance of the authentication and billing system this paper proposed. TJISE has an outstanding performance in school-enterprise cooperation, it has already cooperated with many domestic or international leading manufacturers, software companies and education training institutions. There are more than 10,000 students obtain the practical education 
here. Lots of students, schools and companies comprise the complicated users group, which need a highly efficient authentication and billing system to support the cooperation between different parts.

For the consider of efficient, security and open issues, TJISE adopts the authentication and billing system solution using radius and $802.1 \mathrm{x}$ protocol and system design uses STRUTS frame and MVC technology so that not only realizes the present system management, billing, security and performance requirements, but also provides a convenient for the future expansion of the system. The TJISE internet system has three main subsystems, identity authentication system, backstage charging main system and billing front management system as below.

Identity authentication: use identity authentication system that based on $802.1 \mathrm{x}$ add Radius in campus. There are several steps in the whole process. The user enter a user name and password, the message is delivered to a switchboard port, when the port is an unauthorized state for this user. Then the message is marked PVID at this port and send to corresponding domain to do the authentication according to the user name. If the certain domain is configured with radius authentication mode, the switch port will send the message, which is transfer to radius message, to certain authentication and billing management system. If the user name and password exists in the authentication and billing management system, there will be a successful message back to switchboard. Meantime port changes into authorization state to this user. If the user name does not exist or the password is not right, there will be an unsuccessful message back to switchboard, and the port will remain unauthorized state.

Backstage charging main system: this system will add up and aggregate the billing message in database, will do the cost calculation according to predefined calculation rules, will generate the charging bills, will deducted from the prepaid expenses.

Billing front management system: including user group management, user management, billing strategy management, payment management, network query management, user permission management. The user self service module will provides the interface to query pipeline and bills, and modify user information.

\section{References}

[1]Pascal Urien,Guy Pujolle. Security and privacy for the next wireless generation[J]. Int. J. Network Mgmt.,2008,182:.

[2]Ping Ding. Central Manager: A Solution to Avoid Denial Of Service Attacks for Wireless LANs[J]. International Journal of Network Security,2007,41.

[3] Fanbao Liu, Yumeng Feng, Yuan Cao. On the Security of PPPoE Network.[J]. IACR Cryptology ePrint Archive,2011,2011:

[4] Gaurav Sharma. Hash chain-based authentication and billing for fast handoffs in wireless LANs[D]. University of Missouri - Kansas City, 2004.

[5] Qin Liu, Zhi Yingjian, He Lei, Ming Qing. Reserch on 802.1x Protocol and Its Security Analysis. Computer Engineering. 2007,07:153-154+157. 